\title{
Mélanomes malins: présentation de 3 cas
}

\author{
Haitami $\mathrm{S}^{1}$, Kinani L ${ }^{1}$, Benyahya $\mathrm{I}^{1}$, Badre $\mathrm{L}^{2}$ \\ ${ }^{1}$ Service d'Odontologie chirurgicale, Faculté de Médecine dentaire, Casablanca, Maroc \\ ${ }^{2}$ Laboratoire d'Anatomopathologie privé
}

Le mélanome malin résulte de la transformation maligne des mélanocytes, cellules pigmentaires, qui sont habituellement localisés dans l'épiderme, le derme ou la muqueuse et plus rarement dans les viscères ( Duflo et al. 2008). Les mélanomes buccaux ne représentent, selon les études, que 0,4 à $8 \%$ de l'ensemble de ces tumeurs. Ils occupent néanmoins le $2^{\text {ème }}$ rang des localisations ORL. Malgré cette relative rareté, leur importante malignité et leur pronostic plus sombre que celui des mélanomes cutanés leur confèrent un intérêt certain. Ces tumeurs apparaissent le plus souvent lors de la $5^{\text {ème }}$ et la $6^{\text {ème }}$ décennies. Elles siègent habituellement sur la muqueuse palatine, la fibromuqueuse gingivale, plus rarement sur les lèvres, les joues ou la langue (Femiano et al. 2008). L'étiopathogénie des mélanomes n'est pas totalement élucidée, cependant l'exposition solaire, des facteurs génétiques ou encore la dégénérescence d'un naevus préexistant ont été incriminés ((Kuffer et al. 2009).

Le premier cas rapporté est celui d'une femme âgée de 70 ans ayant consulté pour une tumeur maxillaire indolore, apparue après à une extraction dentaire. Il existe une asymétrie faciale importante ainsi qu'une occlusion oculaire homolatérale. La palpation révèle la présence d'adénopathies submandibulaires homolatérales. L'examen endobuccal montre une tumeur bourgeonnante, noire, s'étendant sur l'ensemble de l'hémi-maxillaire gauche. A l'examen radiologique, on découvre une lyse osseuse importante. La prise en charge de cette patiente a consisté, dans un premier temps, à réaliser une hémi-maxillectomie.

Le second cas est celui d'un homme de 84 ans qui a également consulté pour une asymétrie faciale. L'examen clinique et radiologique a montré une tumeur ayant le même aspect que la précédente, avec une lyse osseuse moins importante. Le traitement a comporté une résection chirurgicale avec mise en place d'une prothèse obturatrice.

Le troisième cas concerne un homme âgé de 50 ans qui s'est présenté pour une lésion plane de couleur foncée, «noir goudron », qui siégeait sur la gencive mandibulaire vestibulaire, puis qui s'est étendue sur la muqueuse linguale. L'examen tomodensitométrique a montré un épaississement des parties molles, sans envahissement osseux associé. La biopsie de la lésion a conclu à un mélanome malin ulcéré. Le patient a été traité par une chirurgie conservatrice associée à une radiothérapie.

Avec ces 3 cas, nous voudrions insister sur la nécessité d'évoquer le diagnostic de mélanome malin devant toute tumeur pigmentée de la peau ou des muqueuses, que celle-ci soit bourgeonnante, ulcérée ou pas. La tumeur peut être le siège d'une pigmentation allant du brun clair au noir anthracite. Le mélanome buccal est souvent découvert tardivement malgré le peu de difficultés pour accéder à cette région, et il a déjà envahi le chorion profond, les tissus mous et l'os sous-jacents, le pronostic n'en est alors que plus mauvais avec un taux de survie dépassant rarement 20\% à 5 ans (Emmanouil et al. 2006).

Correspondance : haitamisofia@yahoo.com 